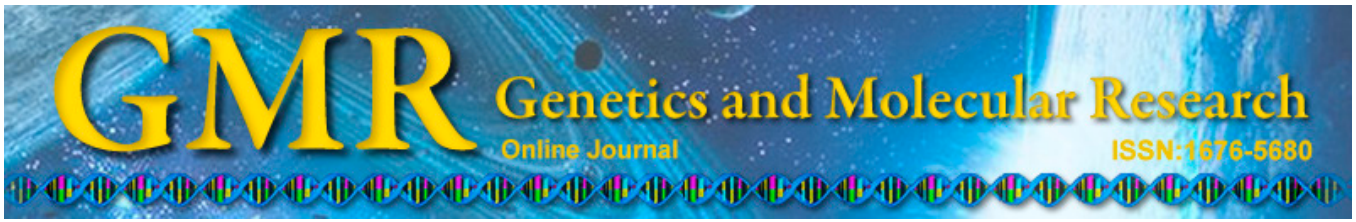

\title{
Differentially expressed genes in the liver of lean and fat chickens
}

\author{
Q. He ${ }^{1,2,3}$, S.Z. Wang ${ }^{1,2,3}$, L. Leng ${ }^{1,2,3}$, W. Na ${ }^{1,2,3}$, Q.G. Wang ${ }^{1,2,4}$ and H. Li ${ }^{1,2,3}$
}

${ }^{1}$ Key Laboratory of Chicken Genetics and Breeding, Ministry of Agriculture, Harbin, China

${ }^{2}$ Key Laboratory of Animal Genetics, Breeding and Reproduction, Education Department of Heilongjiang Province, Harbin, China

${ }^{3}$ College of Animal Science and Technology,

Northeast Agricultural University, Harbin, China

${ }^{4}$ Chongqing Academy of Animal Science, China

Corresponding authors: H. Li / Q.G. Wang

E-mail: lihui@neau.edu.cn / wangqigui@hotmail.com

Genet. Mol. Res. 13 (4): 10823-10828 (2014)

Received January 3, 2014

Accepted June 21, 2014

Published December 19, 2014

DOI http://dx.doi.org/10.4238/2014.December.19.3

\begin{abstract}
This study aimed to investigate gene expression in the chicken liver for lean and fat broiler lines. Birds used in this study were 2 and 4 weeks of age; they were derived from the 14th generation of Northeast Agricultural University broiler lines, which were divergently selected based on abdominal fat content. Chicken Genome Arrays were used to screen differentially expressed genes in the liver tissue from lean and fat birds. At 2 and 4 weeks of age, 770 and 452 genes were differentially expressed between the 2 lines, respectively. The differentially expressed genes were involved in Wnt, insulin signaling,
\end{abstract}


and cell cycle pathways. At 2 and 4 weeks, 42 shared, differentially expressed genes were revealed by the analysis. We speculate that these genes might regulate chicken lipid metabolism.

Key words: Chicken; Lean and fat broiler lines; Chicken liver; Gene expression profile

\section{INTRODUCTION}

Chickens, widely raised farm animals, are excellent animal models for genetic selection/evolutionary research studies. The excessive accumulation of lipids in the adipose tissue of chickens is a major problem in the broiler industry. Unlike mammals, little or no fatty acid synthesis occurs in chicken adipose tissue; the liver is the main tissue of fatty acid synthesis in chickens (Griffin et al., 1992; Cui et al., 2012).

In our previous study, Chicken Genome Arrays were developed to construct gene expression profiles and screen differentially expressed genes in the liver of lean and fat broiler lines at different developmental stages. Our results indicate that 4 weeks of age is a more important stage for chicken liver lipogenesis, and abdominal fat weight (AFW) and percentage of abdominal fat (AFP) are significantly different from those at 2 weeks of age (Wang et al., 2010). In order to better understand gene expression in the chicken liver, gene expression profiles of the liver at 2 and 4 weeks of age were investigated. This study will help to elucidate the molecular mechanisms of lipid metabolism in chickens and contribute to related research in other species.

\section{MATERIAL AND METHODS}

\section{Animals}

The birds utilized in the current study were obtained from the 14th generation population of the Northeast Agricultural University broiler lines, which were divergently selected based on abdominal fat content (Guo et al., 2011). Birds were kept in similar environmental conditions and had access to feed and water. The G0 generation of the 2 lines came from the same grandsire line originating from the Arbor Acres breed.

\section{Sample preparation}

Birds were slaughtered at 2 and 4 weeks of age. The 6 birds used in present study were chosen based on AFP: 3 had high AFP and 3 had low AFP. Total RNA was isolated from the livers using the TRIzol reagent (Invitrogen, Carlsbad, CA, USA) and quantified by spectrophotometry. Messenger RNA (mRNA) was isolated using the Oligotex mRNA MiniKit (Qiagen, Valencia, CA, USA). Complementary DNA (cDNA) was prepared by oligodT-primed reverse transcription (Affymetrix, Santa Clara, CA, USA). cRNA probes were prepared using an IVT Labeling Kit (Affymetrix). The microarrays were prepared using the GeneChip ${ }^{\circledR}$ Hybridization, Wash, and Stain Kit (Affymetrix). The Chicken Genome Arrays, 
with comprehensive coverage of $>38,000$ probe sets representing 32,773 transcripts, were created by Affymetrix, Inc.

\section{Statistical analysis}

Differentially expressed genes were identified from normalized data using the significance analysis of microarrays (SAM) algorithm (Tusher et al., 2001) and the $t$-test (Forrester and Ury, 1969); data were obtained using the procedure of SAS. According to the SAM algorithm, differentially expressed genes are identified based on the expression differences among the sample groups and the consistency of those differences; a score is assigned to each gene on the basis of a change in its expression level relative to the standard deviation of repeated measurements for that gene. Differences were considered highly significant at $\mathrm{P}<0.01$ and significant at $\mathrm{P}<0.05$.

\section{RESULTS}

A P value of 0.05 was used as the threshold for significantly different expression levels. At 2 and 4 weeks of age, 770 and 452 genes were differentially expressed between the 2 lines, respectively. The differentially expressed genes were analyzed by Gene Ontology (GO) enrichment and Kyoto Encyclopedia of Genes and Genomes (KEGG) pathways.

The 770 differentially expressed genes at 2 weeks of age were enriched for 4 significant GO terms $(\mathrm{P}<0.05)$, including ribonucleoprotein complex, structural constituent of ribosome, ribonucleoprotein binding, and establishment of RNA localization functional category. They were mainly annotated to 11 significant pathways by KEGG analysis ( $\mathrm{P}<$ $0.05)$, including ribosome, base excision repair, spliceosome, n-glycan biosynthesis, Wnt signaling pathway, Toll-like receptor signaling pathway, SNARE interactions in vesicular transport, histidine metabolism, protein export, amino sugar and nucleotide sugar metabolism, and insulin signaling pathway.

Differentially expressed genes at 4 weeks of age were significantly enriched for only one GO term (extracellular matrix; $\mathrm{P}=0.0581$ ). They were annotated to 9 significant pathways by KEGG analysis, including cell adhesion molecules, spliceosome, tight junction, notch signaling pathway, cell cycle, intestinal immune network for IgA production, nucleotide excision repair, pentose and glucuronate interconversions, and one carbon pool by folate.

Of the differentially expressed genes, 42 were common in birds belonging to the 2 age groups (Table 1). In comparison to fat chickens, 18 genes were upregulated and 16 were downregulated (Table S1) in lean chickens at 2 and 4 weeks of age, 4 genes were upregulated at 2 weeks and downregulated at 4 weeks, and 4 genes were downregulated at 2 weeks and upregulated at 4 weeks. The upregulated/downregulated genes were analyzed by functional classification and KEGG pathway. The 18 upregulated genes were enriched for 2 significant GO terms, deaminase activity and macromolecular complex, and the 16 downregulated genes were enriched for one significant GO term, ribonucleoprotein binding. These common genes were not involved in any significant pathways via KEGG analysis. 
Q. He et al.

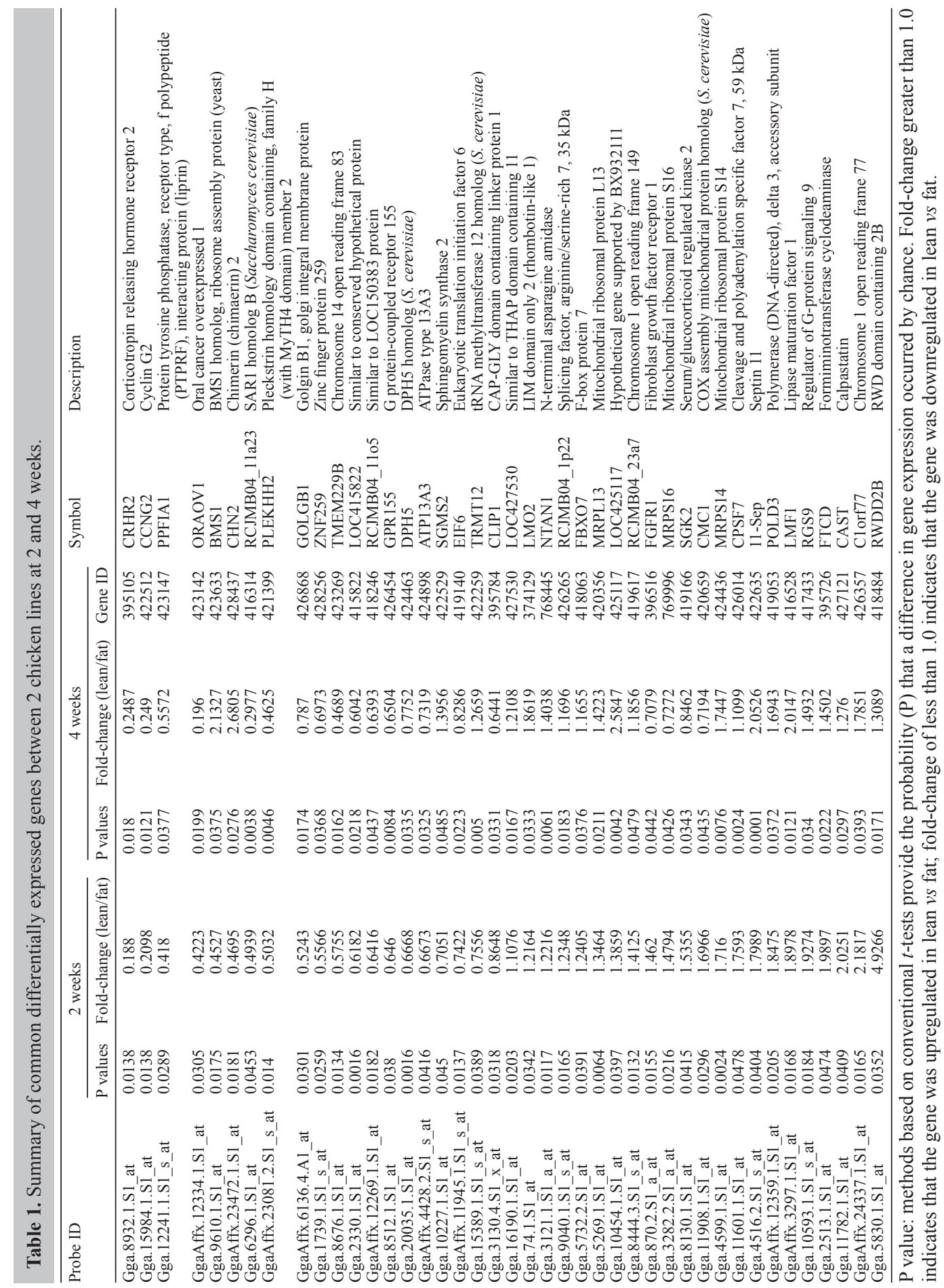




\section{DISCUSSION}

Genome expression analysis provides a broad, unbiased survey of the transcriptome. Bourneuf et al. (2006) identified differentially expressed genes in lean and fat chickens and suggested that the mechanisms of expression and regulation of lipogenic genes could be involved in the ontogenesis of fatness in chickens. In our previous study, we found that the AFW and AFP were significantly different at 2 weeks of age, and 4-week-old broilers displayed the exuberant capability of lipogenesis (Wang et al., 2010). We identified significant pathways and speculated that these pathways, especially the Wnt, insulin signaling, and cell cycle pathways, have important effects on chicken lipid metabolism. The Wnt pathway is essential for the early development of eukaryotic organisms and is involved in cell proliferation, differentiation, and oncogenesis (Kitazoe et al., 2010). Lipid peroxidation products activate the canonical Wnt pathway through oxidative stress (Zhou et al., 2011). The insulin signaling pathway controls the synthesis and accumulation of lipids in the mammalian liver. Insulin-resistant states might lead to abnormal triglyceride deposition in the liver (Leavens and Birnbaum, 2011). Lipids play indispensable roles in cell proliferation, cell differentiation, and organ morphogenesis; these processes are intimately associated with cell cycle progression (Donnelly et al., 1999). Cell cycle progression and neutral lipid turnover appear to be linked (Long et al., 2012). With this in mind, we hypothesize that the 3 pathways play important roles in chicken lipid metabolism.

In summary, our research provided a set of enriched functional pathways and genes that regulate adiposity in chickens, contributing resources to further study the molecular mechanisms of lipid metabolism and fatness variability in lean and fat chicken lines.

\section{ACKNOWLEDGMENTS}

The authors gratefully acknowledge the members of the Poultry Breeding Group of the College of Animal Science \& Technology, Northeast Agricultural University, for help managing the birds and collecting data. Research supported by the National Natural Science Foundation of China (\#30972087), the National "863" Project of China (\#2011AA100301), the China Agriculture Research System (\#CARS-42), and the Program for Innovation Research Team in University of Heilongjiang Province (\#2010td02).

\section{Supplementary material}

\section{REFERENCES}

Bourneuf E, Hérault F, Chicault C, Carre W, et al. (2006). Microarray analysis of differential gene expression in the liver of lean and fat chickens. Gene 372: 162-170.

Cui HX, Zheng MQ, Liu RR, Zhao GP, et al. (2012). Liver dominant expression of fatty acid synthase (FAS) gene in two chicken breeds during intramuscular-fat development. Mol. Biol. Rep. 39: 3479-3484.

Donnelly PM, Bonetta D, Tsukaya H, Dengler RE, et al. (1999). Cell cycling and cell enlargement in developing leaves of Arabidopsis. Dev. Biol. 215: 407-419.

Forrester JC and Ury HK (1969). The signed-rank (Wilcoxon) test in the rapid analysis of biological data. Lancet 1: 239-241.

Griffin HD, Guo K, Windsor D and Butterwith SC (1992). Adipose tissue lipogenesis and fat deposition in leaner broiler chickens. J. Nutr. 122: 363-368. 
Guo L, Sun B, Shang Z, Leng L, et al. (2011). Comparison of adipose tissue cellularity in chicken lines divergently selected for fatness. Poult. Sci .90: 2024-2034.

Kitazoe M, Futami J, Nishikawa M, Yamada H, et al. (2010). Polyethylenimine-cationized beta-catenin protein transduction activates the Wnt canonical signaling pathway more effectively than cationic lipid-based transduction. Biotechnol. J. 5: 385-392.

Leavens KF and Birnbaum MJ (2011). Insulin signaling to hepatic lipid metabolism in health and disease. Crit. Rev. Biochem. Mol. Biol. 46: 200-215.

Long AP, Manneschmidt AK, VerBrugge B, Dortch MR, et al. (2012). Lipid droplet de novo formation and fission are linked to the cell cycle in fission yeast. Traffic 13: 705-714.

Tusher VG, Tibshirani R and Chu G (2001). Significance analysis of microarrays applied to the ionizing radiation response. Proc. Natl. Acad. Sci. U S A 98: 5116-5121.

Wang HB, Wang QG, Zhang XY, Gu XF, et al. (2010). Microarray analysis of genes differentially expressed in the liver of lean and fat chickens. Animal 4: 513-522.

Zhou T, Zhou KK, Lee K, Gao G, et al. (2011). The role of lipid peroxidation products and oxidative stress in activation of the canonical wingless-type MMTV integration site (WNT) pathway in a rat model of diabetic retinopathy. Diabetologia 54: 459-468. 